Abstract P113 Table 1 Patient demographics, disease profile and Quantiferon results

\begin{tabular}{|c|c|c|c|c|c|c|c|}
\hline & All & Male & Female & \multicolumn{4}{|c|}{ Abbreviations used: } \\
\hline $\mathrm{N}$ & 181 & 87 & 94 & \multicolumn{4}{|c|}{ CD Crohn's disease; UC Ulcerative colitis; RA Rheumatoid arthritis; PS Psoriasis \pm arthropathy; N numbe } \\
\hline \multirow[t]{3}{*}{ mean age } & 44.8 & 43.7 & 45.9 & & & & \\
\hline & & & & \multicolumn{4}{|c|}{ Quantiferon result (\%) } \\
\hline & & & & non-reactive & reactive & indeterminate & not done \\
\hline Disease & $\mathrm{N}(\%)$ & & & $95(73.6 \%)$ & $8(6.2 \%)$ & $26(20.2 \%)$ & $52(28.7 \%)$ \\
\hline CD & $78(43.1)$ & 38 & 40 & 48 & 4 & 7 & 19 \\
\hline UC & $65(35.9)$ & 38 & 27 & 33 & 3 & 19 & 10 \\
\hline RA & $20(11.1)$ & 3 & 17 & 7 & 0 & 0 & 13 \\
\hline PS & $14(7.7)$ & 6 & 8 & 6 & 1 & 0 & 7 \\
\hline \multirow[t]{3}{*}{ Other } & $4(2.20$ & 2 & 2 & 1 & 0 & 0 & 3 \\
\hline & & & & \multicolumn{4}{|c|}{ Quantiferon result (\%) } \\
\hline & & & & non-reactive & reactive & indeterminate & \\
\hline \multirow{2}{*}{\multicolumn{3}{|c|}{ For 114 colitis patients - was rescue anti-TNF given? }} & No & 61 & 5 & 6 & \\
\hline & & & Yes & 20 & 2 & $20(p<0.0001)$ & \\
\hline
\end{tabular}

Results 181 patients (M:F 87:94; age range 11-83; average age 44.8 years) had received at least one dose anti-TNF and were included in this study (see Table). The majority of patients had colitis $(\mathrm{n}=143 ; 79 \%) .52$ patients $(28.7 \%)$ did not have a documented QFT or IGRA (interferon gamma release assay) result. LTBI was diagnosed in $8(6.2 \%)$ with reactive QFT of whom 7 completed chemoprophylaxis and 1 was referred for further investigation and treatment elsewhere. 95 (73.6\%) were QFT non-reactive. 26 (20.2\%) were QFT indeterminate, all of whom had a diagnosis of colitis. Colitis patients were more likely to be QFT indeterminate if they were tested at the time of rescue antiTNF with or without surgery (RR 5.71; 95\% CI: 2.49-13.09; $\mathrm{p}<0.0001) .2$ patients who received rescue anti-TNF and were subsequently found to be QFT reactive, successfully completed LTBI chemoprophylaxis.

Conclusion The rate of indeterminate QFT results is higher than expected in our cohort of patients with colitis who require rescue anti-TNF therapy and is likely to be related to the timing of testing. If QFT testing is undertaken, this should be performed when patients are at stable state and not at the time of inflammatory crisis.

\section{P114 IMPLICATIONS OF NICE 2016 TUBERCULOSIS GUIDANCE FOR A TB CONTACT SCREENING SERVICE}

${ }^{1}$ AM Ray, ${ }^{1} S$ Oglesby, ${ }^{2} \mathrm{C}$ Mullarkey, ${ }^{1} \mathrm{JP}$ Watson. 'Leeds Teaching Hospitals NHS Trust, Leeds, UK; ${ }^{2}$ Leeds Community Healthcare NHS Trust, Leeds, UK

\subsection{6/thoraxjnl-2016-209333.257}

Introduction NICE 2016 Tuberculosis guidance recommends significant changes in contact screening. Tuberculin Skin Test (TST) is advocated for diagnosis of latent tuberculosis infection (LTBI), with a positive TST redefined as $5 \mathrm{~mm}$ regardless of BCG status, IGRA only to be used in diagnostic uncertainty, upper age for LTBI treatment raised from 35 to 65 , and contact tracing no longer recommended for extra-pulmonary TB.

We use a 2 step test, with IGRA for those with TST $>10 \mathrm{~mm}$ in context of BCG, and treat LTBI on basis of IGRA result. We aimed to assess the implications for our service of adopting the new guidance.
Methods We reviewed written and electronic records for all contacts screened in Leeds in 2015. NICE 2016 guidance was applied retrospectively to analyse the impact of each recommendation and the guidance as a whole.

Results 216 contacts were screened. Full records were available for 193. 14 were treated for LTBI, 2 for active TB, and 6 contacts over 35 had X-ray follow up. 34 had TST $>10 \mathrm{~mm}$, an additional 13 had TST 5-9 mm. Of 34 with TST > $10 \mathrm{~mm}, 14$ (41\%) had positive IGRA. 97/193 (50\%) were contacts of extrapulmonary tuberculosis. 4 of these were treated for LTBI, but 21 had TST $>5 \mathrm{~mm}$.

Using TST $>5 \mathrm{~mm}$ cut off would increase the number of IGRA tests from 34 to 46 . Treating on basis of TST alone would increase the number given chemoprophylaxis from 14 to 46 . Stopping screening for contacts of extrapulmonary cases would reduce the number screened by $50 \%$ and the number treated from 46 to 29 . However, this would be at the cost of missing at least 4/14 LTBI with positive IGRA.

Conclusion Adopting the new NICE guidance in full would reduce the number screened but significantly increase the numbers treated for LTBI. Using the 2 step test with a TST cut off of $5 \mathrm{~mm}$ would modestly increase the number of IGRA tests but would be unlikely to have a large impact on the number treated. Stopping screening for contacts of extrapulmonary TB would reduce the screening workload by $50 \%$ but reduce the number of LTBI cases diagnosed by $29 \%$.

\section{P115 SCREENING OUTCOMES OF HOUSEHOLD CONTACTS OF MULTIDRUG-RESISTANT TUBERCULOSIS PATIENTS IN PESHAWAR, PAKISTAN}

${ }^{1}$ A Javid, 'MA Khan, ${ }^{2}$ MA Khan, 'S Mehreen, ${ }^{1}$ A Basit, ${ }^{3}$ RA Khan, ${ }^{1}$ M Ihtesham, I I Ullah, ${ }^{1}$ A Khan, ${ }^{1} \mathrm{U}$ Ullah. ${ }^{1}$ Post Graduate Medical Institute, Lady Reading Hospital, Peshawar, Pakistan; ${ }^{2}$ Peshawar Medical College, Peshawar, Pakistan; ${ }^{3}$ Kohat University of Science and Technology, Kohat, Pakistan

10.1136/thoraxjn-2016-209333.258

Objective To assess the profile of $\mathrm{TB} /$ multidrug-resistant $\mathrm{TB}$ (MDR-TB) among household contacts of MDR-TB patients. 
Methods Close contacts of MDR-TB patients were traced in the cross-sectional study. Different clinical, radiological and bacteriological were performed to rule out the evidence of TB/MDR-TB. Results Between January 2012 and December 2012, a total of 200 index MDR-TB patients were initiated on MDR-TB treatment, out of which home visit and contacts screening were conducted for 154 index cases. Of 610 contacts who could be studied, $41(17.4 \%)$ were diagnosed with MDR-TB and 10 (4.2\%) had TB. The most common symptoms observed were cough, chest pain and fever.

Conclusions The high incidence of MDR-TB among close contacts emphasise the need for effective contact screening programme of index MDR-TB cases in order to cut the chain of transmission of this disease.

\section{REFERENCES}

1 WHO. Multidrug resistant TB (MDR-TB): 2014 updated global report. Geneva: World Health OrganisationOrganization, 2014.

2 WHO. Global tuberculosis report 2015. Geneva: World Health OrganisationOrganization, 2015.

3 Khan MA, Mehreen S, Basit A, Khan RA, Javaid A. Predictors of poor outcomes among patients treated for multidrug-resistant tuberculosis at Tertiary Care Hospital in Pakistan. American-Eurasian J Toxicol Sci 2015;7(3):162-172.

\section{P116 MULTIDRUG-RESISTANT TUBERCULOSIS (MDR-TB) MONITORING IN SOUTHEAST LONDON USING CURRENT RECOMMENDATIONS; DOES IT PREVENT COMPLICATIONS?}

${ }^{1}$ B Bradley, ${ }^{1} \mathrm{M}$ Kamalanathan, ${ }^{1} \mathrm{AJ}$ Shah, ${ }^{1} \mathrm{~N}$ Read, ${ }^{1} \mathrm{~J}$ Hall, ${ }^{2} \mathrm{M}$ Belton, ${ }^{1} \mathrm{~L}$ Baker. ${ }^{1}$ Respiratory Department, University Hospital Lewisham, Lewisham and Greenwich NHS Trust, London, UK; ${ }^{2}$ Queen Elizabeth Hospital, Lewisham and Greenwich NHS Foundation Trust, London, UK

\subsection{6/thoraxjnl-2016-209333.259}

Background MDR-TB requires an intense treatment course with multiple drugs, many of which have significant recognised side effects. Frequency of monitoring to prevent long term complications is not fully established, but recent guidelines have gone some way to addressing this. In light of this we wanted to review the side effects of MDR-TB treatment in our population to identify whether our previous practice was in line with the current recommendations and if not, whether the currently recommended frequency could have identified these complications sooner.

Methods 26 patients referred to our trust over 13 years (between 2002 and 2015) had a diagnosis of MDR-TB on a basis of isoniazid and rifampicin resistance on culture and/or PCR. Medical records were reviewed; diagnostic tests, resistance profiles, baseline investigations, treatment, drug monitoring tests and side effects were recorded on 21 of these patients. These were compared with current European Respiratory Society guidelines for MDR-TB management.

Results All baseline tests were completed except for magnesium and electrocardiogram. 6 patients had care transferred elsewhere. Amikacin/cycloserine levels were performed in line with recommendations. Interval blood testing was not always undertaken as recommended, particularly near the end of treatment. 11/15 (73\%) of patients experienced at least 1 complication to treatment. Most frequent were: amikacin induced ototoxicity in $5 / 15$ (33\%), PAS/prothionamide induced hypothyroidism in $3 / 15$ (20\%). Other complications included photosensitivity with pyrazinamide, clofazimine associated erythema, peripheral neuropathy secondary to linezolid/cycloserine, amikacin induced renal impairment and ureteric colic secondary to calculi. Audiometry revealed high frequency hearing loss prior to development of symptoms. One patient developed ototoxicity despite monthly audiometry testing. Hypothyroidism developed despite monthly thyroid function.

Conclusions In our population there was a high incidence of significant side effects to MDR-TB regimes. Ototoxicity with amikacin is a significant concern. While frequent testing is advocated we found that complications were not necessarily negated by this. Frequency of testing was easier to achieve whilst an inpatient than an outpatient. With the advent of the World Health Organisation shorter MDR-TB regimen, the need for regular monitoring remains crucial, but the shorter duration of injectable drugs may also help decrease complication rates in the future.

\section{P117 PHARMACY-LED LATENT TB INFECTION SERVICE: A SUCCESS STORY}

YO Abunga, M Day, SO Brij. Peterborough City Hospital, Peterborough, UK

\subsection{6/thoraxinl-2016-209333.260}

Introduction Previously we have reported good outcomes following a pilot study of latent TB infection (LTBI) assessment and treatment by a dedicated TB Pharmacist. ${ }^{1}$ Since that time, the service has been imbedded within our local practice to reduce waiting times, improve chemoprophylaxis adherence, enhance treatment outcomes, and minimise adverse drug reactions whilst maintaining a high standard of care. In addition, patients with more complex medical needs requiring biological therapy are now not excluded from this service.

Aim to review the safety and effectiveness of the Pharmacy-led Clinic

Methods Medical records of all cases of LTBI seen by the TB Pharmacist were reviewed retrospectively. Data obtained included patient demographics, treatment \pm side-effects and clinical outcomes.

Results 206 patients with LTBI seen between Jan 2012 and December 2015 were identified (see Table 1). Contact tracing and the requirement for biological therapy were the commonest reasons for referral. $185(89.8 \%)$ successfully completed treatment: 3 declined; 8 lost to follow up; 10 stopped due to sideeffects. Significant drug side-effects included hepatitis, peripheral neuropathy, nausea and vomiting and only resulted in 2 noncompletion, the rest were due to fatigue and headache. Of the 8 who stopped 1 developed active TB whilst on biological therapy. Patient satisfaction was good and patients would recommend this service to a friend or family member.

Conclusion The LTBI Pharmacy-led clinic has been successfully implemented and incorporated into our local TB practice with good outcomes. Keeping with the Carter Review, ${ }^{2}$ it is important to utilise the skills and knowledge of previously unrecognised clinical partners to deliver high quality care. Patients were happy to be seen by the pharmacists. Poly pharmacy patients appreciated time spent to simplify regime and manage potential interaction.

\section{REFERENCES}

1 Abunga YO, Luwemba G, Brij SO. Diagnosis and management of TB: P59 Treatment and Drug Surveillance of Latent Tuberculosis Infections (LTBI) by a TB Pharmacist: A pilot study. Thorax 2012;67(Suppl 2):A88.

2 Department of Health. Independent report - Productivity in NHS hospitals [ONLINE]. https://www.gov.uk/government/uploads/system/uploads/attachment_data/file/499229/Operational_productivity_A.pdf (Accessed 6 July 2016). 